\title{
A Regiospecific One-Pot, Three Component Synthesis of 4-Aryl-6,8-dimethylpyrimido[4,5-c]pyridazine-5,7(6H,8H)-diones as New Potential Monoamine Oxidase Inhibitors
}

\author{
Jabbar Khalafy, ${ }^{*}$ Mehdi Rimaz, Leila Panahi, and Hossein Rabiei \\ Chemistry Department, Urmia University, P.O. Box 57154, Urmia, Iran \\ E-mail: jkhalafi@yahoo.com; j.khalafi@mail.urmia.ac.ir \\ Received May 10, 2011, Accepted June 3, 2011
}

\begin{abstract}
A series of new 4-aryl-6,8-dimethylpyrimido[4,5-c]pyridazine-5,7(6H,8H)-diones have been synthesized via three component reaction of 1,3-dimethylbarbituric acid with arylglyoxals in the presence of hydrazinium dihydrochloride in ethanol. All of these derivatives may act as potential monoamine oxidase inhibitors.
\end{abstract}

Key Words : Pyrimido[4,5-c]pyridazine, Monoamine, Arylglyoxal, Barbituric acid

\section{Introduction}

A major challenge of modern drug discovery is the design of highly efficient chemical reaction sequences that provide maximum structural complexity and diversity with a minimum number of synthetic steps to assemble compounds with interesting properties. ${ }^{1}$ Recently multicomponent reactions (MCRs) have emerged as a highly valuable tool in modern organic synthesis and modern drug discovery. The atom economy and convergent character, ${ }^{2}$ the simplicity of a one-pot procedure, the possible structural variations, the accessible complexity of the molecules, and the very large number of accessible compounds are among the described advantages of a MCR. ${ }^{3}$

The exploration of privileged structures in drug discovery is a rapidly emerging theme in medicinal chemistry. ${ }^{4}$ The pyridazine structural framework and heterofused analogues as important privileged heterocyclic scaffolds continue to attract attention due to their wide variety of interesting biological activities. ${ }^{5}$ The synthesis and utility of many pyridazine derivatives as analgesics, insecticidals, ${ }^{6}$ fungicidals ${ }^{7,8}$ cardiotonics, ${ }^{9}$ and bacteriocides ${ }^{10}$ have been reported. In particular, pyrimido[4,5-c]pyridazine-5,7(6H,8H)diones are common sources for the development of new potential therapeutic agents. ${ }^{11-13}$ Carotti and coworkers have demonstrated that the 3-arylpyrimido[4,5-c]pyridazine5,7 $(6 H, 8 H)$-diones 1-2 have MAO inhibitory activity, and substituents on the diazine nucleus modulate the inhibitory activity. ${ }^{14}$

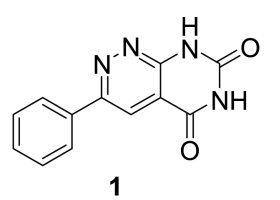<smiles>O=c1[nH]c(=O)c2cc(-c3ccc(C(F)(F)F)cc3)nnc2[nH]1</smiles>

Monoamine oxidase (MAO) is a ubiquitous membranebound, avin-containing enzyme, which is particularly abundant in the liver and brain. ${ }^{15}$ MAO is located intracellularly in the mitochondrial outer membranes of neuronal, glial, and other cells and catalyzes the oxidative deamination of monoamine neurotransmitters both from endogenous and exogenous sources to the corresponding aldehydes with consumption of oxygen and production of hydrogen peroxide, thereby affecting the concentrations of neurotransmitter amines as well as many xenobiotic ones. ${ }^{16-18}$ In mammals, two distinct isoforms of MAO are present in most tissues, namely, MAO-A and MAO-B, which were defined in 1968, based on their differential substrate and inhibitor specicity, ${ }^{19-21}$ tissue and cell distribution, ${ }^{22}$ and gene expression $^{23,24}$ characteristics. Recognition of the importance of monoamine oxidases as targets for drug intervention for treatment of a variety of conditions has produced an enormous interest in development of inhibitors of these enzymes during these years.

In continuation of our research interests regarding the development of the synthetic utility of arylglyoxals as valuable sources, ${ }^{25-28}$ we wish to report herein a simple and convenient protocol for regiospecific synthesis of 4-aryl-6,8dimethylpyrimido[4,5-c]pyridazine-5,7(6H,8H)-diones 3 via a one-pot three-component tandem [2+2] annulation of 1,3-
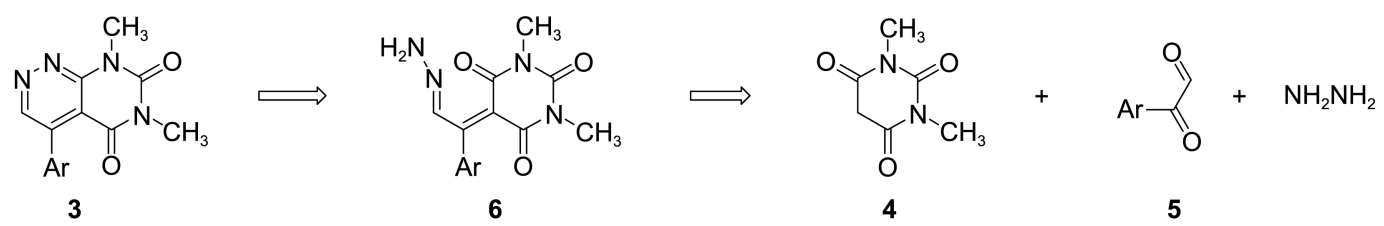

Scheme 1. Retrosynthetic analysis for 4-aryl-6,8-dimethylpyrimido[4,5-c]pyridazine-5,7(6H,8H)-dione. 
dimethylbarbituric acid $\mathbf{4}$ with arylglyoxals 5 in the presence of hydrazinium dihydrochloride (Scheme 1). To the best of our knowledge, there have not been reports about the regiospecific synthesis of 4-aryl substituted pyrimidopyridazine scaffold via a MCR method.

\section{Results and Discussion}

Arylglyoxals 17-26 were prepared from the oxidation of

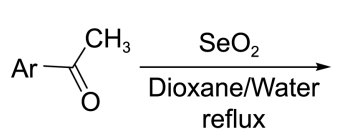<smiles>O=CC(=O)Br</smiles>

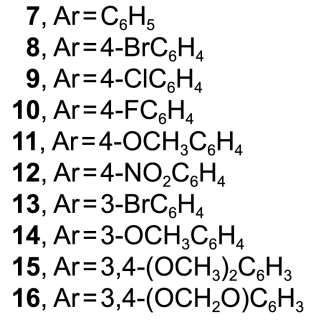

17, $\mathrm{Ar}=\mathrm{C}_{6} \mathrm{H}_{5}$

18, $\mathrm{Ar}=4-\mathrm{BrC}_{6} \mathrm{H}_{4}$

19, $\mathrm{Ar}=4-\mathrm{ClC}_{6} \mathrm{H}_{4}$

20, $\mathrm{Ar}=4-\mathrm{FC}_{6} \mathrm{H}_{4}$

21, $\mathrm{Ar}=4-\mathrm{OCH}_{3} \mathrm{C}_{6} \mathrm{H}_{4}$

22, $\mathrm{Ar}=4-\mathrm{NO}_{2} \mathrm{C}_{6} \mathrm{H}$

23, $\mathrm{Ar}=3-\mathrm{BrC}_{6} \mathrm{H}_{4}$

24, $\mathrm{Ar}=3-\mathrm{OCH}_{3} \mathrm{C}_{6} \mathrm{H}_{4}$

25, $\mathrm{Ar}=3,4-\left(\mathrm{OCH}_{3}\right)_{2} \mathrm{C}_{6} \mathrm{H}_{3}$

26, $\mathrm{Ar}=3,4-\left(\mathrm{OCH}_{2} \mathrm{O}\right) \mathrm{C}_{6} \mathrm{H}_{3}$

Scheme 2. Synthesis of arylglyoxals.

Table 1. Synthesis of 4-aryl-6,8-dimethylpyrimido[4,5-c]pyridazine-5,7 $(6 H, 8 H)$-dione

Entry Arylglyoxal Pyrimidopyridazine Yield (\%)

their corresponding acetophenones 7-16 using $\mathrm{SeO}_{2}$ as shown in Scheme 2. ${ }^{29}$

Ten examples of the arylglyoxals conversion to various 4aryl substituted pyrimidopyridazine systems are listed in Table 1 . The reactions were performed by adding arylglyoxals 5 to 1,3-dimethyl barbituric acid 4 in the presence of stoichiometric amount of hydrazinium dihydrochloride salt in refluxing ethanol (Scheme 3). After 12 hours, the precipitate was collected by filtering. The products were

Table 1. Continued

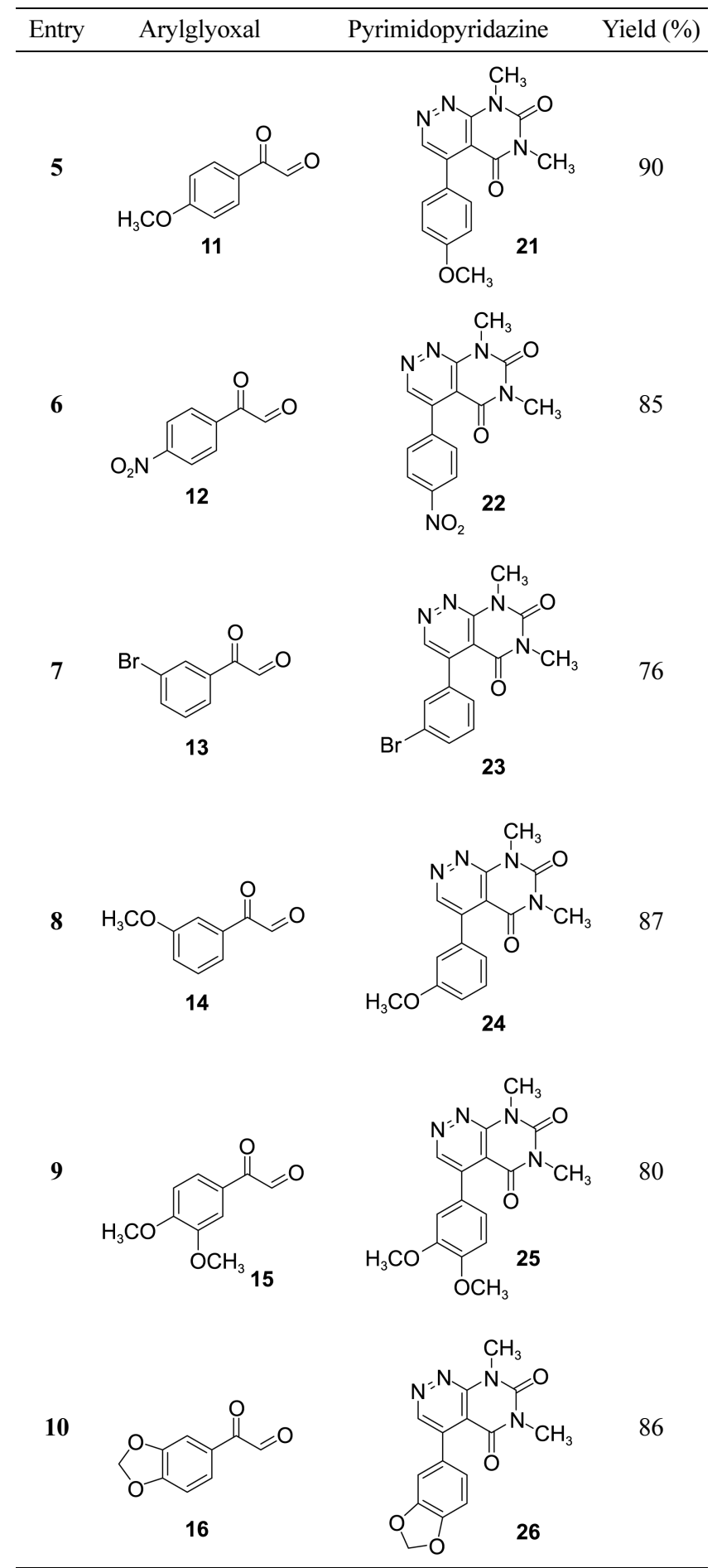



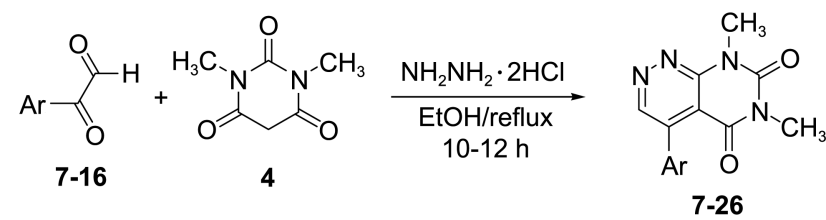

Scheme 3. Synthesis of 4-aryl-6,8-dimethylpyrimido[4,5-c]pyridazine-5,7 $(6 H, 8 H)$-dione.

obtained in good yields after recrystallization in methanol.

The suggested mechanism for regiospecific formation of 4-aryl substituted pyrimidopyridazine $\mathbf{2 6}$ as single product involves the initial formation of arylglyoxal monohydrazone 27 through a simple imine condensation reaction and the subsequent knoevenagel condensation of 1,3-dimethyl barbituric acid enolic form $\mathbf{2 8}$ with the aryl monohydrazone carbonyl leads to form the intermediate 29. Progress of the reaction via fulfillment of an intramolecular imine condensation in the intermediate $\mathbf{2 9}$ gave the final product $\mathbf{2 6}$ in $86 \%$ yield (Scheme 4$)$.

In order to elucidation of the proposed mechanism and proving this fact that all of the obtained products are only 4aryl substituted isomers and there is no evidence for existence of 3-aryl substituted ones, we prepared the aryl monohydrazones separately and reacted them with 1,3dimethyl barbituric acid under the same reaction conditions. All of the spectral data and physical properties of the products obtained from this two stages strategy and the products of the one-pot strategy were identical.

\section{Experimental}

General Procedures. Freshly distilled solvents were used throughout, and anhydrous solvents were dried according to Perrin and Armarego. ${ }^{30}$ Melting points were determined on a Philip Harris C4954718 apparatus. Infrared spectra were recorded on a Thermonicolet (Nexus 670) Fourier-transform (FT) infrared spectrometer, using $\mathrm{KBr}$ discs. ${ }^{1} \mathrm{H}$ (300 MHz) and ${ }^{13} \mathrm{C}(75.5 \mathrm{MHz}) \mathrm{nmr}$ measurements were recorded on a Bruker 300 spectrometer in $\mathrm{CDCl}_{3}$ using TMS as the internal reference. Mass spectra were recorded on a Varian Matt 311 spectrometer and relative abundances of fragments are quoted in parentheses after the $\mathrm{m} / \mathrm{z}$ values. Microanalyses were performed on a Leco Analyzer 932.

General Procedure for the Synthesis of 4-aryl-6,8-dimethylpyrimido[4,5-c]pyridazine-5,7(6H,8H)-diones. A mixture of 1,3-dimethylbarbituric acid $(1 \mathrm{mmol})$ and arylglyoxal $(1 \mathrm{mmol})$ in the presence of hydrazinium dihydrochloride $(1 \mathrm{mmol})$ in absolute ethanol $(10 \mathrm{~mL})$ was refluxed for 12 hours. The obtained precipitation was separated by filteration and washed with excess rectified spirit. Recrystalization from absolute methanol gave the title compounds as pure products in good yields.

4-Phenyl-6,8-dimethylpyrimido[4,5-c]pyridazine-5,7(6H, 8H)-dione (17): white solid; 90\%; mp $244{ }^{\circ} \mathrm{C}$ (dec.); ${ }^{1} \mathrm{H}-$ NMR $\delta 3.55$ (s, 3H), 3.97 (s, 3H), 7.52-7.61 (m, 3H), 8.16 $\left(\mathrm{dd}, J_{1}=7.2 \mathrm{~Hz}, J_{2}=1.8 \mathrm{~Hz}, 2 \mathrm{H}\right), 8.53(\mathrm{~s}, 1 \mathrm{H}) ;{ }^{13} \mathrm{C}-\mathrm{NMR} \delta$ 28.9, 29.9, 113.7, 122.0, 126.7, 129.3, 130.3, 133.2, 134.9, 150.30, 156.7, 160.6; FT-IR (KBr) $v_{\max } / \mathrm{cm}^{-1}: 1708,1668$, 1604, 1459, 1303, 1002, 792, 695, 482, 420; Mass $m / z(\%)$ : $268\left(\mathrm{M}^{+}, 100\right), 211$ (20), 182 (18), 156 (32), 140 (58), 104 (21), 102 (24); Anal. Calcd for $\mathrm{C}_{14} \mathrm{H}_{12} \mathrm{~N}_{4} \mathrm{O}_{2}$ : C, 62.68; H, 4.51 ; N, 20.88. Found: C, 62.63; H, 4.53; N, 20.78.

4-(4-Bromophenyl)-6,8-dimethylpyrimido[4,5-c]pyridazine-5,7(6H,8H)-dione (18): cream solid; 77\%; mp $296^{\circ} \mathrm{C}$ (dec.); ${ }^{1} \mathrm{H}-\mathrm{NMR} \delta 3.55$ (s, 3H), 3.96 (s, 3H), 7.71 (d, $J=8.7$ $\mathrm{Hz}, 2 \mathrm{H}), 8.02$ (d, $J=8.7 \mathrm{~Hz}, 2 \mathrm{H}), 8.49(\mathrm{~s}, 1 \mathrm{H}) ;{ }^{13} \mathrm{C}-\mathrm{NMR} \delta$ 28.9, 29.9, 113.7, 121.8, 125.1, 128.1, 132.5, 133.9, 150.4, $155.7,157.8,160.5$; FT-IR $(\mathrm{KBr}) v_{\max } / \mathrm{cm}^{-1}: 1718,1670$, 1596, 1449, 1319, 1293, 1075, 1005, 944, 840, 824, 793, 743, 485, 418; Mass $m / z(\%): 348\left[\left(\mathrm{M}^{+}+2\right), 100\right], 346\left[\mathrm{M}^{+}\right.$, (98)], 220 (35), 182 (50), 180 (34), 155 (55), 139 (43), 113 (36), 101 (45), 84 (31), 75 (38), 71 (35), 69 (42), 57 (89), 55 (55), 43 (71), 41 (50); Anal. Calcd for $\mathrm{C}_{14} \mathrm{H}_{11} \mathrm{BrN}_{4} \mathrm{O}_{2} ; \mathrm{C}$, 48.43; H, 3.19; N, 16.14. Found: C, 48.51; H, 3.23; N, 16.20.

4-(4-Chlorophenyl)-6,8-dimethylpyrimido[4,5-c]pyridazine-5,7(6H,8H)-dione (19): white solid; 92\%; mp 277 ${ }^{\circ} \mathrm{C}$ (dec.); ${ }^{1} \mathrm{H}-\mathrm{NMR} \delta 3.55$ (s, 3H), 3.97 (s, 3H), 7.55 (d, $J=$ $8.7 \mathrm{~Hz}, 2 \mathrm{H}), 8.11(\mathrm{~d}, J=8.7 \mathrm{~Hz}, 2 \mathrm{H}), 8.49(\mathrm{~s}, 1 \mathrm{H}) ;{ }^{13} \mathrm{C}-\mathrm{NMR}$ $\delta 28.9,29.9,113.7,121.8,127.9,129.5,133.4,136.8,150.3$,
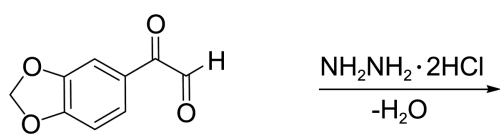

16

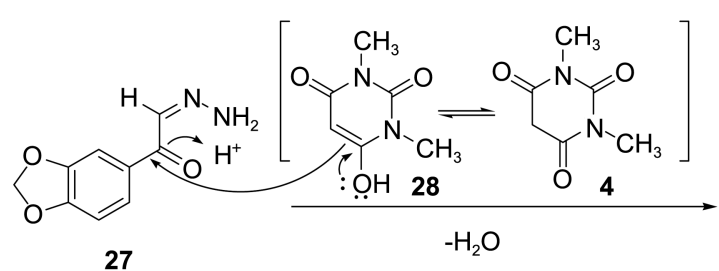

27<smiles>CN1CCCCCCCC(=O)N(C)C(=O)/C(=C(\C=N/N)c2ccc3c(c2)OCO3)C1=O</smiles>
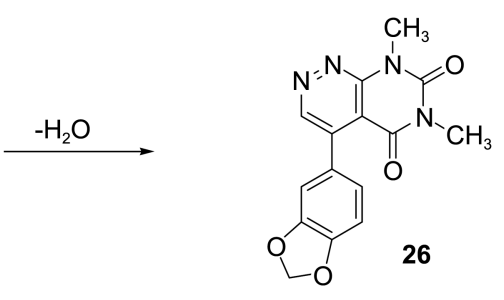

Scheme 4. Suggested mechanism for regiospecific synthesis of 4-aryl-6,8-dimethylpyrimido[4,5-c]pyridazine-5,7(6H,8H)-dione. 
150.4, 155.6, 160.5; FT-IR (KBr) $v_{\max } / \mathrm{cm}^{-1}: 1720,1672$, 1596, 1450, 1321, 1292, 1156, 1093, 1072, 1008, 945, 845, 826, 793, 743, 482, 417; Mass $m / z(\%): 302\left(\mathrm{M}^{+}, 92\right), 245$ (31), 190 (30), 176 (34), 174 (100), 149 (50), 139 (38), 138 (57), 136 (41), 113 (36), 75 (42), 70 (23), 57 (62), 56 (41), 55 (43), 43 (68), 41 (81); Anal. Calcd for $\mathrm{C}_{14} \mathrm{H}_{11} \mathrm{ClN}_{4} \mathrm{O}_{2}$ : C, $55.55 ; \mathrm{H}, 3.66$; N, 18.51. Found: C, 55.64; H, 3.61; N, 18.39 .

4-(4-Fluorophenyl)-6,8-dimethylpyrimido[4,5-c]pyridazine-5,7(6H,8H)-dione (20): white solid; 80\%; mp 229 ${ }^{\circ} \mathrm{C}$ (dec.); ${ }^{1} \mathrm{H}-\mathrm{NMR} \delta 3.54$ (s, 3H), 3.95 (s, 3H), 7.25 (t, $J=$ $8.1 \mathrm{~Hz}, 2 \mathrm{H}), 8.12-8.17(\mathrm{~m}, 2 \mathrm{H}), 8.46(\mathrm{~s}, 1 \mathrm{H}) ;{ }^{13} \mathrm{C}-\mathrm{NMR} \delta$ 28.9, 29.9, 113.7, 116.2, 116.5, 121.7, 128.6, 128.7, 131.1, $131.1,150.2,150.3,155.7,160.50,162.6,165.9$; FT-IR $(\mathrm{KBr}) v_{\max } / \mathrm{cm}^{-1}: 1718,1665,1598,1471,1445,1291,1225$, 1158, 1003, 856, 743, 484, 422; Mass $m / z(\%): 286\left(\mathrm{M}^{+}, 22\right)$, 229 (25), 174 (26), 158 (100), 131 (32), 120 (36), 81 (24), 57 (34), 55 (30), 43 (26), 41 (27); Anal. Calcd for $\mathrm{C}_{14} \mathrm{H}_{11} \mathrm{FN}_{4} \mathrm{O}_{2}$ : C, 58.74; H, 3.87; N, 19.57. Found: C, 58.82; H, 3.89; N, 19.50 .

4-(4-Methoxyphenyl)-6,8-dimethylpyrimido[4,5-c]pyridazine-5,7(6H,8H)-dione (21): white solid; 90\%; mp 246 ${ }^{\circ} \mathrm{C}$ (dec.); ${ }^{1} \mathrm{H}-\mathrm{NMR} \delta 3.54$ (s, 3H), 3.91 (s, 3H), 3.94 (s, 3H), 7.08 (d, $J=8.4 \mathrm{~Hz}, 2 \mathrm{H}), 8.12$ (d, $J=8.4 \mathrm{~Hz}, 2 \mathrm{H}), 8.45$ (s, $1 \mathrm{H}) ;{ }^{13} \mathrm{C}-\mathrm{NMR} \delta 28.8,29.9,55.4,113.8,114.7,121.4,127.3$, $128.1,149.8,150.3,156.3,160.6,161.6$; FT-IR $(\mathrm{KBr}) v_{\max } /$ $\mathrm{cm}^{-1}: 1714,1668,1606,1518,1458,1439,1404,1287$, 1252, 1176, 1038, 1018, 1002, 834, 792, 747, 539, 485, 421; Mass $m / z(\%): 298\left(\mathrm{M}^{+}, 46\right), 241$ (62), 227 (40), 170 (65), 127 (43), 89 (39), 81(33), 69 (61), 57 (77), 56 (40), 55 (59), 43 (100), 41 (98); Anal. Calcd for $\mathrm{C}_{15} \mathrm{H}_{14} \mathrm{~N}_{4} \mathrm{O}_{3}: \mathrm{C}, 60.40 ; \mathrm{H}$, 4.73 ; N, 18.78. Found: C, 60.49; H, 4.79; N, 18.63.

4-(4-Nitrophenyl)-6,8-dimethylpyrimido[4,5-c]pyridazine-5,7(6H,8H)-dione (22): gray solid; $85 \%$; mp $278{ }^{\circ} \mathrm{C}$ (dec.); ${ }^{1} \mathrm{H}-\mathrm{NMR} \delta 3.57$ (s, 3H), 3.99 (s, 3H), 8.36 (d, $J=8.4$ $\mathrm{Hz}, 2 \mathrm{H}), 8.4(\mathrm{~d}, J=8.4 \mathrm{~Hz}, 2 \mathrm{H}), 8.6(\mathrm{~s}, 1 \mathrm{H}) ;{ }^{13} \mathrm{C}-\mathrm{NMR} \delta$ 28.9, 29.9, 123.1, 124.6, 126.4, 128.3, 128.8, 141.9, 149.0, 153.9, 156.6, 160.7; FT-IR (KBr) $v_{\max } / \mathrm{cm}^{-1}: 1723,1673$, 1602, 1517, 1450, 1344, 1292, 1148, 1108, 853, 728, 690, 475; Mass $m / z(\%): 313\left(\mathrm{M}^{+}, 6\right), 302$ (100), 190 (35), 174 (51), 155 (31), 138 (38), 136 (28), 81 (28), 69 (43), 57 (31), 43 (34), 41 (31); Anal.Calcd for $\mathrm{C}_{14} \mathrm{H}_{11} \mathrm{~N}_{5} \mathrm{O}_{4}$ : C, 53.68; H, 3.54; N, 22.36. Found: C, 53.72; H, 3.51; N, 22.27.

4-(3-Bromophenyl)-6,8-dimethylpyrimido[4,5-c]pyridazine-5,7(6H,8H)-dione (23): cream solid; 76\%; mp 211 ${ }^{\circ} \mathrm{C}$ (dec.); ${ }^{1} \mathrm{H}-\mathrm{NMR} \delta 3.55$ (s, 3H), 3.96 (s, 3H), 7.44 (t, $J=$ $7.8 \mathrm{~Hz}, 1 \mathrm{H}), 7.67$ (d, $J=7.8 \mathrm{~Hz}, 1 \mathrm{H}), 8.07$ (d, $J=7.5 \mathrm{~Hz}$, 1H), 8.35 (bs, 1H), 8.49 (s, 1H); ${ }^{13} \mathrm{C}-\mathrm{NMR} \delta 28.9,29.9$, $113.6,122.1,123.5,125.2,129.8,130.7,133.2,136.9$, 150.3, 150.6, 155.2, 160.4; FT-IR (KBr) $v_{\max } / \mathrm{cm}^{-1}: 1715$, 1667, 1592, 1456, 1291, 1214, 1161, 999, 796, 737, 683, 487, 418; Mass $m / z(\%): 348$ [( $\left.\left.\mathrm{M}^{+}+2\right), 100\right], 346\left(\mathrm{M}^{+}, 95\right)$, 220 (35), 218 (54), 155 (67), 139 (46), 113 (35), 101 (30), 75 (33), 63 (28), 43 (28); Anal. Calcd for $\mathrm{C}_{14} \mathrm{H}_{11} \mathrm{BrN}_{4} \mathrm{O}_{2}$ : C, 48.43; H, 3.19; N, 16.14. Found: C, 48.49; H, 3.24; N, 16.02 .

4-(3-Methoxyphenyl)-6,8-dimethylpyrimido[4,5-c]pyridazine-5,7(6H,8H)-dione (24): cream solid; 87\%; mp 223 ${ }^{\circ} \mathrm{C}$ (dec.); ${ }^{1} \mathrm{H}-\mathrm{NMR} \delta 3.53$ (s, 3H), 3.92 (s, 3H), 3.95 (s, 3H), $7.07(\mathrm{~d}, J=8.4 \mathrm{~Hz}, 1 \mathrm{H}), 7.46(\mathrm{t}, J=8.1 \mathrm{~Hz}, 1 \mathrm{H}), 7.64(\mathrm{~d}, J=$ $7.8 \mathrm{~Hz}, 1 \mathrm{H}), 7.78$ (bs, 1H), $8.50(\mathrm{~s}, 1 \mathrm{H}) ;{ }^{13} \mathrm{C}-\mathrm{NMR} \delta 28.9$, 29.9, 30.9, 55.5, 111.3, 113.6, 116.7, 118.9, 122.2, 130.2, $136.2,150.3,156.3,160.4,160.5$; FT-IR $(\mathrm{KBr}) v_{\max } / \mathrm{cm}^{-1}$ : $1717,1667,1596,1471,1452,1326,1289$, 1250, 1172, $1028,1000,874,793,739,687,480,417$; Mass $m / z(\%)$ : $298\left(\mathrm{M}^{+}, 11\right), 268$ (45), 156 (32), 140 (100), 113 (39), 102 (39), 57 (47), 51 (30), 43 (46), 41 (49); Anal. Calcd for $\mathrm{C}_{15} \mathrm{H}_{14} \mathrm{~N}_{4} \mathrm{O}_{3}$ : C, 60.40; H, 4.73; N, 18.78. Found: C, 60.46; $\mathrm{H}, 4.77$; N, 18.85 .

4-(3,4-Dimethoxyphenyl)-6,8-dimethylpyrimido[4,5-c]pyridazine-5,7(6H,8H)-dione (25): cream solid; $80 \%$; mp $283{ }^{\circ} \mathrm{C}$ (dec.); ${ }^{1} \mathrm{H}-\mathrm{NMR} \delta 3.54$ (s, 3H), 3.94 (s, 3H), 3.97 (s, $3 \mathrm{H}), 4.02(\mathrm{~s}, 3 \mathrm{H}), 7.01(\mathrm{~d}, J=8.4 \mathrm{~Hz}, 1 \mathrm{H}), 7.60(\mathrm{~d}, J=8.4$ $\mathrm{Hz}, 1 \mathrm{H}), 7.88$ (s, 1H), $8.46(\mathrm{~s}, 1 \mathrm{H}) ;{ }^{13} \mathrm{C}-\mathrm{NMR} \delta 28.9,29.9$, 56.0, 56.1, 109.3, 111.3, 113.8, 119.5, 121.6, 127.5, 149.8, $149.9,150.3,151.3,156.1,160.6$; FT-IR $(\mathrm{KBr}) v_{\max } / \mathrm{cm}^{-1}$ : 1719, 1670, 1518, 1458, 1295, 1268, 1156, 1020, 870, 740, 480; Mass $m / z(\%): 328\left(\mathrm{M}^{+}, 100\right), 327$ (21), 313 (15), 285 (16), 271 (17), 257 (14), 83 (16), 57 (18), 55 (17), 43 (16); Anal. Calcd for $\mathrm{C}_{16} \mathrm{H}_{16} \mathrm{~N}_{4} \mathrm{O}_{4}$ : C, 58.53; H, 4.91; N, 17.06 . Found: C, 58.63; H, 4.97; N, 17.00.

4-(Benzo $[d][1,3]$ dioxol-5-yl)-6,8-dimethylpyrimido $[4,5-$ clpyridazine-5,7(6H,8H)-dione (26): cream solid; $86 \%$; mp $256{ }^{\circ} \mathrm{C}$ (dec.); ${ }^{1} \mathrm{H}-\mathrm{NMR} \delta 3.54$ (s, 3H), 3.94 (s, 3H), 6.09 (s, 2H), $6.98(\mathrm{~d}, J=8.1 \mathrm{~Hz}, 1 \mathrm{H}), 7.61(\mathrm{~d}, J=8.1 \mathrm{~Hz}, 1 \mathrm{H})$, $7.72(\mathrm{~s}, 1 \mathrm{H}), 8.43(\mathrm{~s}, 1 \mathrm{H}) ;{ }^{13} \mathrm{C}-\mathrm{NMR} \delta 28.9,29.9,101.7$, $106.9,108.9$, 113.7, 121.1, 121.6, 129.1, 148.8, 149.7, $149.9,150.3,156.2,160.6$; FT-IR $(\mathrm{KBr}) v_{\max } / \mathrm{cm}^{-1}: 1714$, 1672, 1597, 1455, 1358, 1296, 1249, 1151, 1040, 930, 866, 836, 738, 418; Mass m/z (\%): $312\left(\mathrm{M}^{+}, 68\right), 284$ (42), 256 (23), 255 (100), 241 (26), 57 (20), 43 (32), 41 (27); Anal. Calcd for $\mathrm{C}_{15} \mathrm{H}_{12} \mathrm{~N}_{4} \mathrm{O}_{4}$ : C, 57.69; H, 3.87; N, 17.94. Found, C, 57.73; H, 3.90; N, 17.83 .

\section{Conclusion}

The one-pot strategy outlined provides a very potent straightforward route for regiospecific synthesis of various 4-aryl-6,8-dimethylpyrimido[4,5-c]pyridazine-5,7(6H,8H)diones. Moreover, the compounds are currently being evaluated as monoamine oxidase inhibitors.

Acknowledgments. The authors express their thanks to Urmia University for financial support.

\section{References}

1. (a) Schreiber, S. L. Science 2000, 287, 1964. (b) Zhu, J.-P.; Bienaymé, H. Multicomponent Reactions; Wiley-VCH: Weinheim, 2005; p 1499. (c) Trost, B. M. Angew. Chem., Int. Ed. Engl. 1995, 34, 259. (d) Tietze, L. F. Chem. Rev. 1996, 96, 115.

2. (a) Dömling, A.; Ugi, I. Angew. Chem., Int. Ed. 2000, 39, 3168. (b) Dömling, A. Chem. Rev. 2006, 106, 17. (c) Diego, J. R.; Miguel, Y. Angew. Chem., Int. Ed. 2005, 44, 1602. (d) Tietze, L. F.; Brasche, G.; Gericke, K. M. Domino Reaction in Organic Synthesis; Wiely-VCH: Weinheim, 2006; p 542. (e) James, D. S.; Chris, D.; Stephen, F. M. Org. Lett. 2007, 9, 4223.

3. (a) Ganem, B. Acc. Chem. Res. 2009, 42, 463. (b) Barry, B. T.; 
Dennis, G. H. Chem. Rev. 2009, 109, 4439. (c) Zhou, H. Y.; Zhang, W.; Yan, B. J. Comb. Chem. 2010, 12, 206. (d) Liu, A. F.; Zhou, H. Y.; Su, G. X.; Zhang, W.; Yan, B. J. Comb. Chem. 2009, 11, 1083. (e) Jiang, B.; Tu, S.-J.; Kaur, P.; Wever, W.; Li, G.-G. J. Am. Chem. Soc. 2009, 131, 11660. (f) Jiang, B.; Wang, X.; Shi, F.; Tu, S.-J.; Ai, T.; Ballew, A.; Li, G. G. J. Org. Chem. 2009, 74, 9486. (g) Wei, H.-L.; Yan, Z.-Y.; Niu, Y.-L.; Li, G.-Q.; Liang, Y.M. J. Org. Chem. 2007, 72, 8600. (h) Cui, S.-L.; Wang, J.; Wang, Y.-G. Org. Lett. 2008, 10, 13. (i) Cui, S.-L.; Wang, J.; Lin, X.-F.; Wang, Y.-G. J. Org. Chem. 2007, 72, 7779. (j) Cao, H.; Wang, X.J.; Jiang, H.-F.; Zhu, Q.-H.; Zhang, M.; Liu, H.-Y. Chem.-Eur. J. 2008, 14, 11623. (k) Zhang, M.; Jiang, H.-F.; Liu, H.-L .; Zhu, Q.H. Org. Lett. 2007, 9, 4111. (1) Liu, W.-B.; Jiang, H.-F.; Huang, L.B. Org. Lett. 2010, 12, 312. (m) Yan, C.-G.; Wang, Q.-F.; Song, X.-K.; Sun, J. J. Org. Chem. 2009, 74, 710. (n) Wang, Q.-F.; Hou, H.; Hui, L.; Yan, C.-G. J. Org. Chem. 2009, 74, 7403. (o) Sun, J.; Zhang, L.-L.; Xia, E.-Y.; Yan, C.-G. J. Org. Chem. 2009, 74, 3398.

4. (a) Douglas, A. H.; Gregory, T. B.; Mark, L. S. Chem. Rev. 2003, 103, 893. (b) Poupaert, J.; Carato, P.; Colacino, E. Curr. Med. Chem. 2005, 12, 877. (c) Triggle, D. J. Cell. Mol. Neurobiol. 2003, 23, 293. (d) Bemis, G. W.; Murcko, M. A. J. Med. Chem. 1996, 39, 2887.

5. Quintela, J. M.; Veiga, M. C.; Conde, S.; Peinador, C. Monatsh. Chem. 1996, 127, 739 .

6. Numata, T.; Ogura, T.; Hirat, K.; Kudo, M. Jpn Kokai Tokyo Koho Jp. 1989, 63, 159, 372 [Chem. Abstr. 1989, 110, 75538].

7. Yoshioka, H.; Obato, T.; Fujii, K.; Fukuda, Y.; Ooka, A. Eur. Pat. Appl. Ep. 1989, 283, 271.

8. Matolesy, G. World Rev Pest Contr, 1971, 10, 50 [Chem. Abstr. 1972, 76, 820315].

9. Okujima, H.; Naeimatsu, A.; Kobayashi, M.; Funlya, R.; Kitada, K. Jpn Kokai Tokyo Jp. 1989, 63, 215, 672 [Chem. Abstr. 1989, 110,75541].

10. Preshin, G. N.; Sherbakova, L. I.; Zykova, T. N.; Sokolova, V. N. Farmakol Tokisikol. 1971, 35, 466 [Chem. Abstr. 1972, 77, 1355802].

11. Brown, D. J. In Comprehensive Heterocyclic Chemistry; Katritzky, A. R., Rees, C. W. Eds.; Pergamon Press: Oxford, 1984; Vol. 3, p
57.

12. Wamhoff, H.; Dzenis, J.; Hirota, K. Adv. Heterocycl. Chem. 1992, $55,129$.

13. Hamilton, G. A. in Progress in Bioorganic Chemistry; Kaiser, E. T., Kezdy, F. J., Eds.; Wiley: New York, 1971; Vol. 1, p 83.

14. Altomare, C.; Cellamare, S.; Summo, L.; Catto, M.; Carotti, A. J. Med. Chem. 1998, 41, 3812.

15. Kalgutkar, A. S.; Dalvie, D. K.; Castagnoli, N., Jr.; Taylor, T. J. Chem. Res. Toxicol. 2001, 14, 1139.

16. Haung, R. H.; Faulkner, R. J. Biol. Chem. 1981, 256, 9211.

17. Cohen, G.; Farooqui, R.; Kesler, N. Proc. Natl. Acad. Sci. U.S.A. 1997, 94, 4890 .

18. Edmondson, D. E.; Mattevi, A.; Binda, C.; Li, M.; Hubálek, F. Curr. Med. Chem. 2004, 19, 1983.

19. Youdim, M. B. H.; Finberg, J. P. M. Biochem. Pharmacol. 1991, $41,155$.

20. Gottowik, J.; Cesura, A. M.; Malherbe, P.; Lang, G.; Prada, M. D. FEBS Lett. 1993, 317, 152.

21. Geha, R. M.; Rebrin, I.; Chen, K.; Shih, J. C. J. Biol. Chem. 2001, 276, 9877.

22. Westlund, K. N.; Denney, R. M.; Kochersperger, L. M.; Rose, R. M.; Abell, C. W. Science 1985, 230, 181.

23. Bach, A. W. J.; Lan, N. C.; Johnson, D. L.; Abell, C. W.; Bembenek, M. E.; Kwan, S. W.; Seeburg, P. H.; Shih, J. C. Proc. Natl. Acad. Sci. U.S.A. 1988, 85, 4934.

24. Grimsby, J.; Chen, K.; Wang, L. J.; Lan, N. C.; Shin, J. C. Proc. Natl. Acad. Sci. U.S.A. 1991, 88, 3637.

25. Rimaz, M.; Khalafy, J. Arkivoc 2010, (ii), 110

26. Rimaz, M.; Khalafy, J.; Najafi Moghadam, P. Aust. J. Chem. 2010, 63, 1396.

27. Rimaz, M.; Khalafy, J.; Noroozi Pesyan, N.; Prager, R. H. Aust. J. Chem. 2010, 63, 507.

28. Rimaz, M.; Noroozi Pesyan, N.; Khalafy, J. Magn. Reson. Chem. 2010, 48, 276.

29. Riley, H. A.; Gray, A. R. Organic Syntheses; Wiley \& Sons: New York, NY, 1943; Collect. Vol. II, p 509.

30. Perrin, D. D.; Armarego, W. L. F. Purification of Laboratory Chemicals; Pergamon Press: Oxford, U.K., 1988. 